Man and Nature

L'homme et la nature

\title{
Aux armes et aux Arts! La société populaire et républicaine des arts et le Journal de Détournelle
}

\section{Claudette Hould}

Volume 10, 1991

URI : https://id.erudit.org/iderudit/1012622ar

DOI : https://doi.org/10.7202/1012622ar

Aller au sommaire du numéro

Éditeur(s)

Canadian Society for Eighteenth-Century Studies / Société canadienne d'étude du dix-huitième siècle

ISSN

0824-3298 (imprimé)

1927-8810 (numérique)

Découvrir la revue

Citer cet article

Hould, C. (1991). Aux armes et aux Arts! La société populaire et républicaine des arts et le Journal de Détournelle. Man and Nature / L'homme et la nature, 10, 47-56. https://doi.org/10.7202/1012622ar

Copyright (c) Canadian Society for Eighteenth-Century Studies / Sociéte canadienne d'étude du dix-huitième siècle, 1991
Ce document est protégé par la loi sur le droit d'auteur. L'utilisation des services d’Érudit (y compris la reproduction) est assujettie à sa politique d'utilisation que vous pouvez consulter en ligne.

https://apropos.erudit.org/fr/usagers/politique-dutilisation/ 


\section{Aux armes et aux Arts! La société populaire et républicaine des arts et le Journal de Détournelle}

Dans sa séance du 6 Pluviôse an II (25 janvier 1794), l'architecte Détournelle lit aux membres de la Société Populaire et Républicaine des Arts le prospectus d'un journal, relatif aux beaux-arts, qu'il se propose de rédiger en son nom. L'assemblée accueille favorablement l'introduction qu'il leur soumet et lui donne accès à ses archives et procès-verbaux. Le titre du journal Aux armes et aux arts! $!^{1}$ reflète exactement le rapport entre l'art et le politique en France sous la Terreur, l'idéologie militariste des artistes 'patriotes' et la prépondérance du militaire sur l'art; il restitue le contenu des débats animés, souvent violents ou partisans, non seulement au sein de la Société Populaire et Républicaine des arts mais également dans les séances du Jury des arts de février 1794 poursuivis dans le Club Révolutionnaire des Arts, à partir du 1er mars. Complément utile aux procès-verbaux de la Commune des Arts et de la Société, ${ }^{2}$ le Journal de Détournelle constitue un des plus précieux documents de la période révolutionnaire pour saisir la pensée esthétique et politiqne des artistes et mesurer l'ampleur de leur engagement dans la Révolution.

Ce 6 Pluviôse (25 janvier 1794) donc, c'est la onzième réunion consignée dans les procès-verbaux de la nouvelle Société, 'épurée,' qui avait d'abord été constituée le 18 juillet précédent sous le nom de Commune générale des Arts. ${ }^{3}$ Implicitement, la Commune devait alors remplacer l'Académie de peinture et de sculpture supprimée officiellement avec toutes les Académies, le 8 août 1793. Après quatre mois de travaux, la Commune des Arts, accusée de continuer le système académique, avait été abolie par un décret de la Convention. ${ }^{4}$

Curieusement, la page de titre du journal Aux armes et aux arts! le fait commencer au 1er Ventôse (19 février) alors que Détournelle rend compte des séances à partir du 3 Pluviôse $(22 \text { janvier })^{5}$ en les faisant précéder d'une cinquantaine de pages de documents divers concernant les séances du premier mois consigné dans les procès-verbaux de la Société Populaire et Républicaine des arts, ainsi dénommée pour la première fois le 3 Nivôse ( 23 décembre) ${ }^{6}$ 
C'est par le Journal, puisqu'ils sont absents des procès-verbaux, que nous connaissons les règlements de la nouvelle formation: Contrairement à la Commune 'où l'on recevait indistinctement tous les artistes,' la SPRA décida se s'épurer: 'un noyau de Patriotes connus fut formé; un creuset épuratoire fut chauffé, le feu ardent, dont on l'entretient sans cesse, écarte les faux patriotes. ${ }^{7}$

Un comité de présentation de 4 membres et un comité d'épuration de 8 membres président à l'admission des candidats, qui doivent montrer leur carte de Citoyen (certificat de civisme) et justifier de leur exercice dans la Garde nationale et de leur contribution patriotique, après quoi le Président les interpelle ainsi:

As-tu signé quelque pétition ou fait quelque écrits anti-civiques? as-tuété Membre d'aucun Club proscrit par l'opinion publique? as-tu signé ou accepté la Constitution décrétée par la Convention Nationale?

Les règlements stipulent que le fait d'exposer publiquement des ouvrages contraires au respect dû aux bonnes moeurs et la publication d'écrits anti-civiques sont des 'motifs de réjection.'

La carte d'entrée est inspectée par le censeur à chaque séance et reçoit une marque différente à tous les trimestres.

La cotisation comprend un droit d'admission de 50 sols + 3 livres par trimestre ( 20 sols par mois) payables à l'avance.

Ces règlements démontrent à eux seuls la rigidité idéologique de la Société et témoignent de l'esprit de censure qui semblait prévaloir dans cette assemblée: les dénonciations, les menaces et les exclusions de membres rapportées tant dans les procès-verbaux que dans le Journal sont nombreuses et la fameuse dénonciation de Wicar et de ses comparses contre les artistes français restés en Italie demeure sans doute l'une des pages les plus noires de l'histoire de l'art durant la Révolution française.

A propos de l'épuration de la Société, il existe deux listes de membres dont la comparaison est éloquente: la première liste, manuscrite aux Archives nationales, ${ }^{9}$ a été livrée à sa demande au Comité d'Instruction publique le 4 novembre 1793 après la dissolution de la Commune des arts. Elle comprend 324 noms de peintres, 99 noms de sculpteurs, 114 noms d'architectes et 97 noms de graveurs, au total 634 artistes. La seconde, nous la trouvons sur le plat inférieur des couvertures du Journal de Détournelle qui imprime une vingtaine de noms 'par ordre d'épuration et de réception' dans cinq numéros (les deux premiers numéros se répètent) soit un total de 99 artistes. L'interruption de la publication du Journal ne lui a pas permis de nommer les membres reçus après le 3 
Pluviôse, consignés dans les procès-verbaux, mais l'écart est assez significatif pour imaginer le climat de terreur régnant. La mention d'un ordre de réception indique en outre que les 60 premiers noms ont été reçus au cours des mois de novembre et de décembre 1793 pendant les séances dont il ne nous est parvenu aucun des procès-verbaux. A titre d'exemple, je ne citerai ici que les cas de Regnault et Boilly: le peintre académicien Regnault qui, bien 'qu'admis depuis longtemps' est présenté à la société le 19 Nivôse (8 janvier) mais il en est exclu dans la séance suivante, le 23 (12 janvier), parce qui'il a été soi-disant inculpé par David dans son rapport sur la suppression de la Commission du Museum. La SPRA demande qu'il se procure un certificat de civisme du Comité révolutionnaire de sa Section. ${ }^{10}$

Quant à Boilly, ayant été dénoncé dans une des Séances comme l'auteur de gravures indécentes, il dut se présenter à la séance du 9 Floréal (28 avril) pour se justifier et assurer [...] 'que jamais il n'a dicté les titres qui sont au bas des estampes, qu'il est le premier à les dénoncer, que ses tableaux ont été composés bien avant la révolution, et qu'il a expié les erreurs $d^{\prime}$ une composition un peu libre, en exerçant son pinceau d'une manière plus digne des Arts. ${ }^{11}$ Il s'agit évidemment ici d'une estampe licencieuse intitulée $\mathrm{Ca}$ ira, qui montre un couple enlaçé et des seins dénudés. Invité à consacrer son génie à la Révolution et à faire reconnaître au Comité d'organisation les pièces propres à autoriser sa réception dans la Société, il dut probablement prouver son civisme en produisant son Triomphe de Marat après son acquittement par le Tribunal Révolutionnaire (Musée des beaux-arts de Lille: ' 'L'Assemblée satisfaite des raisons que le $C$. Boille [a abjuré ses anciennes erreur] a communiques a l'assemblée arrête qu'il se présentera au Committéd'organisation pour y faire reconnaître les pièces propre a autoriser sa reception dans la Societté'). ${ }^{13}$ Le 29 Floréal an II (18 mai 1794) Boilly était admis au sein de la Société. ${ }^{14}$

Le cas cité de Boilly est un excellent exemple de la complémentarité du Journal et des procès-verbaux: sur certains points ces derniers sont plus complets, mais dans l'ensemble Détournelle fournit des versions plus circonstanciées des faits et des discussions.

Dans le cadre restreint de ce très court exposé, j'ai choisi de rendre compte d'une partie du Journal, totalement exclue des procès-verbaux, les compte-rendus des Séances du Jury chargé de juger le concours de 1793. Les délibérations rapportées en détail par Détournelle sont une des rares sources pour comprendre le comportement des artistes durant la Révolution et surtout leur attitude devant les doctrines esthétiques défendues par les tenants de l'Antiquité auxquels s'opposaient à la fois les Révolutionnaires purs et durs et les tenants de la doctrine libérale.

Composé de 50 membres, le jury était formé d'artistes, d'hommes de lettres, acteurs, hommes politiques et même d'un jardinier et d'un 
cordonnier; il s'agissait en effet du premier jugement exercé complètement en dehors de l'Académie, puisque la moitié des membres du jury du concours de 1791 étaient d'office académiciens.

Ce jury devait juger le Concours pour les prix de sculpture, peinture et architecture, décrété les 9 et 25 Brumaire ( 30 oct. et 15 nov. 1793). Les oeuvres avaient été exposées publiquement au Museum des Arts pendant 5 jours à partir du 28 janvier et le jury commença à se réunir le 7 février pour juger $d$ 'abord la sculpture en bas-relief pour lequel quatre concurrents avaient été retenus. Il va sans dire que les sujets étaient empruntés à l'histoire antique romaine.

\section{Programme de sculpture:}

Le maître d'Ecole de Falairies renvoyé dans cette ville par Camille, à qui ce traître avoit voulu livrer ses disciples.

Le moment est celui où les pères et mères des jeunes Falisques viennent au-devant de leurs enfans qui ramènent leur Maitre d'Ecole en le fustigeant. $2 \mathrm{e}$ volume de l'Histoire romaine, par Rolin.

Il ne me semble pas inutile d'éclairer le contexte des délibérations en précisant que le président élu du jury était Pache, le maire de Paris, et qu'un des secrétaires était Fleuriot, le substitut à l'accusateur public, Fouquier-Tinville au Tribunal révolutionnaire. C'est donc Fleuriot qui pose au départ pour critère de jugement 'que tous les concurrents mettront à côté de leurs talens ce qu'ils auront fait pour la Révolution. ${ }^{15}$

L'interrogation de Fleuriot contient une menace:

Avant de juger, il faut savoir quel caractère ont pris les Arts; ensuite, s'il est révolutionnaire, et si les Artistes en sont pénétrés; il y en a au combat, aux frontières; je me suis demandé pourquoi il en restoit ici d'oisifs pour la Révolution, et qui s'occupoient à faire des bas-reliefs. Je n'ai pas considéré l'ouvrage en lui-même; j'ai cherché à voir ses rapports avec l'intérêt de la République, et je ne l'ai pas rencontré. Celui qui est resté, étoit-il utile, oui ou non, au mouvement de la Révolution? J'ai vu des jeunes gens travaillant au métier de la Sculpture, et j'en ai vu peu qui avoient imprégnés leurs bas-reliefs, du génie que fomente les grands principes révolutionnaires; j'ai vu des hommes qui s'occupoient de la Sculpture, pendant que leurs frères versoient leur sang por la patrie: mon opinion est et sera pour la négative. ${ }^{16}$

Malgré l'insistance de Dufourny pour dire 'qu'ils doivent ici juger les talens et non les personnes ... et qu'ils sont venus ici récompenser le génie 
et non des ouvriers, ${ }^{17} 40 / 41$ votants décident qu'il n'y a pas lieu de décerner un prix pour la sculpture.

Au plan esthétique, le débat se situe autour de la doctrine académique fondée sur le dessin et la rigueur technique contre l'école du sentiment soutenue par Hassenfradz: 'on ne doit pas juger sur le faire de la main; l'esprit, la passion, le sentiment, voilà ce qui caractérise un chef-d'oeuvre..... ${ }^{18}$

Dans ses réflexions sur cette première séance du jury, Détournelle constate et semble approuver 'l'inflexible sévérité révolutionnaire, jointe à cette vérité, que tout doit céder devant l'expression et le sentiment.' A l'objection que 'dans l'agitation, au milieu du bruit des armes, le génie des Arts ne pouvoit avoir toute sa vigueur,' Détournelle répond que le jury ne pouvait couronner des oeuvres 'qui manquoient de vérité, d'expression et de sentiment.' Son 'opinion est que le Jury a bien jugé la Sculpture,' mais quant à lui il 'aurait voté pour les quatre un encouragement, si les renseignemens sur leur conduite républicaine eussent été satisfaisants.'

Le jugement de la peinture donna lieu à des débats encore plus passionnés, d'autant plus qu'on sent planer l'ombre de David dont c'est d'ailleurs un élève qui remportera le prix.

Programme: Brutus tué dans un combat; les Elèves retraceront le moment où les Chevaliers Romains transporteront son corps à rome, et où les Consuls vont au-devant pour le recevoir.

Les cinq concurrents sont passés au crible et on décide de donner des prix même si les tableaux sont faibles car les sujets sont républicains et traités de manière républicaine. Cette fois la discussion s'allonge pour décider s'il y aura un premier ou un second prix.

Allais précise que les artistes concurrents sont aux frontières en raison de leur âge et qu'il devrait y avoir deux prix. ${ }^{19}$ Il plaide également leur jeune âge pour motiver l'indulgence.

A nouveau, Fleuriot fait porter l'essentiel de la discussion sur le contenu révolutionaire et moral des arts et sur la pertinence de produire des oeuvres d'art en temps de guerre: 'Les productions du génie ne sont utiles qu'autant qu'elles transmettent la vertu.' Il revient sur la question des artistes partis aux armées 'Craignez que les artistes qui se battent ne viennent nous dire, que nous avons récompensé la médiocrité qui est restée ici oisive dans les ateliers; Fleuriot 'distingue celui qui a concourru depuis six mois, et qui est parti par réquisition, d'avec celui qui depuis dix-huit, supporte les fatigues militaires [volontaire]. ${ }^{20}$

La discussion sur le fond met en opposition, comme cela avait été le cas pour la sculpture, 'le sentiment, l'expression, l'âme, la correction; l'originalité qui constitue la physionomie d'un bon tableau et les règles de la géométrie'; 
Hassenfradz provoque les rires et les murmures de l'assemblée, perfidement rendus par Détournelle, en dénonçant l'absence de la perspective dans les tableaux: '... j'ai une intime conviction que tous les objets de Peinture peuvent être faits avec la règle et le compas' (Hassenfradz entame une longue discussion sur le fait qu'il y a plus d'un point de fuite dans le tableau $n^{\circ} 3 .^{21}$ Le Sueur s'élève contre le recours aux valeurs académiques en opposition à Caraffe qui fait l'éloge du dessin. ${ }^{22}$

La majorité prononce qu'il n'y aura qu'un second Prix, accordé à 44/47 voix à Harriet, dont Fleuriot indique le jeune âge, 16 ans et demi, et le fait qu'il est l'élève du Grand-Maître [David].

Cette fois Détournelle fait une cabale contre le gagnant dans ses Réflexions sur le Jugement de Peinture ${ }^{23}$ et établit courageusement son opposition au système académique fondé sur la copie de l'antique et des Maîtres. Il aurait voté pour le $\mathrm{N}^{\circ} 2$ qui n'a obtenu que 3 votes et ' $a$ le défaut d'être trop beau pour l'âge de l'auteur.' Il préfère même le $\mathrm{N}^{\circ} 1$ au $\mathrm{N}^{\circ} 3$. Il explique les critères du jury: en votant pour le $\mathrm{N}^{\circ} 3$ les membres du jury ont eu pour but 'l'expression, le sentiment et très peu de considératon pour le métier.' Il conseille à Harriet 'de s'attacher non à copier l'antique, mais la nature que l'antique a copiée.' Il suggère que certaines de ses figures ont été inspirées par des gravures consultées au Cabinet des estampes. Donc, il semble penser que les tenants du système académique dominent encore, tout en prenant ses distances avec le modèle antique: 'Nous ne serons ni Athéniens, ni Romains; esclaves qui portaient le nom d'hommes libres, mais Français libres par nature, Philosophes par caractère, vertueux par sentiment, et Artistes par goût. ${ }^{24}$ Détournelle félicite tout de même la Convention pour son initiative et le mode du concours.

Lors de la troisième séance du Jury le 19 Pluviôse ( 7 février), Harriet est présenté par le président Dufourny comme le peintre 'qui a plus mérité par l'âme que par le talent de la main.' Il annonce qu'il sera présenté à la Convention avec son tableau. Dufourny remarque que le rédacteur du procès-verbal a mis que le jeune homme était élève de David et s'en inquiète: 'Les méchans pourroient dire que le Maître nous a influencé sur le choix.' Hébert se dit d'avis contraire 'que l'on doit aussi honorer le talent $d u$ Maître....'

C'est la séance du Jugement des prix d'architecture ${ }^{25}$ qui est rapportée le plus longuement par Détournelle, qui, étant lui-même architecte remet en question la présence de profanes et d'artistes autres qu'architectes dans un tel jugement qui, pense-t-il aurait exigé un jury spécial de spécialistes, vu l'impossibilité pour le peuple naïf de juger l'architecture, beaucoup plus difficile à juger que la peinture.

Programme: Une caserne capable de contenir six cents hommes de cavalerie. 
Base de jugement d'après Lussaut: grandeur, convenance.

Des membres outrés de la mauvaise formulation du programme voudraient l'ignorer. ${ }^{26}$ Détournelle s'y oppose en silence, sachant que le concurrent $\mathrm{N}^{\circ} \mathrm{l}$ avait failli abandonner le concours 'pour donner carrière à son génie' mais que tout candidat n'avait pas le choix de se conformer ou non au programme. Ne nous étonnons pas de retrouver à nouveau Fleuriot entonner son credo: il dit devoir ramener les arts à la Révolution, préconise l'art révolutionnaire, opposé au système académique: 'Nous sommes appelés, non à juger un prix, mais à régénérer les Arts.' Il reproche encore aux élèves de n' avoir pas été à l'armée: 'servaient-ils mieux la patrie en faisant un projet d'Architecture, n'était-il pas plus convenable d'aller combattre dans nos armées? non; contens de voir couronner leur amour-propre, ils ont préféré de faire des plans à celui de verser leur sang pour la patrie.'

Fleuriot conclut de ne pas accorder de prix d'architecture. Ce jugement paraît trop sévère à Détournelle qui expose en détail les louanges et les reproches adressés aux concurrents et se permet même des dénonciations: Le Sueur vote pour le prix à son frère [Protin?]; un concurrent triche.

Dans ses Réflexions suivant le Jugement de l'Architecture, Détournelle reproche aux membres du jury leurs absences, considérées comme un manque de civisme: sur 50 membres du jury, 47 votants se sont présentés en Peinture; en Sculpture: 41 votants et en Architecture: 39 votants. Détournelle voudrait même voir annuler le jugement en architecture à cause du petit nombre de votants et suggère d'en appeler auprès de la Convention. Il n'aurait pas donné de prix 'mais, après le jugement, aurait demandé des encouragements aux concurrents qui auraient, depuis la Révolution, fait preuve de patriotisme.' Tout en conseillant aux architectes français de ne pas oublier les lois retenues de l'Antique, il critique l'Antiquomanie en faveur d'un style français.

Lors de la Quatrième Séance du Jury National des Arts, tenuele 20 Pluviôse, avant que de se transporter à la Convention nationale (le 8 février, pour y présenter Harriet et Protin), ${ }^{27}$ le président dit à Protin, gagnant du concours d'architecture:

Ton projet étoit foible; nous avons jugé qu'il ne méritoit qu'un encouragement, cultive les dispositions que tu annonces; les armes que tu portes nous font voir que tu sais allier les travaux guerriers à ceux du génie, continue à servir Mars et Apollon, et voue ton compas à illustrer la Liberté et la République.

A l'issue du jugement, les membres du jury décident de se former en Club révolutionnaire des arts pour discuter des arts et de leur régénéra- 
tion plutôt que de faire admettre les jurés à la SPRA. Fleuriot accuse la Société d'accueillir encore les 'préjugés.'

\section{CONCLUSION}

Sur la couverture du 6e numéro (publiéà la fin de mai 1794) Détournelle fait paraître un AVIS aux souscripteurs, les priant de ne renouveler leur abonnement que lorsqu'ils recevront le numéro 7: 'Quoique foible pour lutter dans les Concours avec les grands talens, le devoir exige que je m'occupe de différens projets, dont le travail m'empêche de déterminer l'époque où paroîtra le prochain numéro.' Le dernier 'Mélanges' du dernier numéro, ${ }^{28}$ évoque la diminution de l'intérêt des citoyens pour les réunions: le 24 Floréal (13 mai) et le 4 Prairial (22 mai), le Club Révolutionnaire des Arts n'a pu tenir de séance, faute de participants. Détournelle annonce également la nomination par le Comité de Salut public, de Fleuriot comme Maire de Paris $[10 \text { mai } 1794]^{29}$ en remplacement de Pache, mis en état d'arrestation. La Terreur repoussait loin de ces éminents membres du Jury des arts les préoccupations artistiques qui les avaient retenus trois mois plus tôt. Fleuriot (vaguement étudiant en architecture et en sculpture, élu substitut à l'accusateur public Fouquier-Tinville au Tribunal révolutionnaire, le 13 mars 1793) sera guillotiné le 28 juillet 1794, le même jour que Robespierre.

\section{CLAUDETTE HOULD}

Maison des étudiants canadiens, Paris

\section{Notes}

1 Aux armes et aux arts Peinture, sculpture, architecture et gravure. Journal de la Société républicaine des arts, séante au Louvre, salle du Laocoon, rédigé par Détournelle, architecte. In $8^{\circ}$. Six numéros en 394 pages (les seuls parus), de l'Imprimerie de Fantelin. s.d. (19 fév.-20 mai 1794). Paris, Bibliothèque nationale, V 42711.

Détournelle, qui a été contesté au sein de la Société pour l'équivoque de son titre homonyme (Lapauze, p. 238), l'a sans doute enrichi au moment de faire relier les six numéros qui composent la première partie, inspiré de toute évidence par une inscription du Muséum dont nous connaissons l'existence par les procès-verbaux officiels de la Société. Dans la séance du 26 Pluviôse an II (14 février 1794) un membre réclame...contre une inscription qui se trouve dans le museum national, et qui porte ces mots, aux arts et aux armes. Le patriotisme, dit-il, devant avoir le pas sur les arts; et les armes étant le soutien du patriotisme, il demande qu'on obtienne ce changement civique; aux armes et aux arts (Lapauze, p. 229). 
2 Lapauze, Henry, Procès-verbaux de la Commune générale des arts de peinture, sculpture, architecture et gravure (18 juillet 1793-tridi de la 1ère décade du deuxième mois de l'An II) et de la société Populaire et républicaine des arts, (3 Nivôse an II-28 Floréal an III), (Paris, Imprimerie nationale, J.-E. Bulloz, éd., 1903).

3 Commune des arts avait également été le nom donné au tout premier regroupement d'artistes en septembre 1790. C'est ce groupe qui avait fini par obtenir l'ouverture du Salon à tous les artistes en 1791 et la suppression de l'Académie de peinture et de sculpture en août 1793. Le 4 juillet 1793, un décret de la Convention confie à six artistes nommés par la Société des Arts tenant ses séances au Louvre, la tâche de préserver les monuments. La Société se réunira le 18 juillet sous le titre de Commune Générale des Arts.

4 Dès les premiers jours de la Commune Générale des Arts David subit une humiliation devant Dardel qui fut élu président à sa place à 50 voix contre $42 \mathrm{au}$ premier tour et à 80 contre 23 au ballotage. Ce seul incident marquait le sort de cette institution.

5 L'erreur est corrigée dans l'AVIS AU LECTEUR. La SPRA se réunissait neuf fois par mois, les 6 et 9 de chaque décade.

6 Infra SPRA. Entre le 24 octobre 1793, date du dernier procès-verbal de la Commune des Arts, et le 23 décembre 1793, date du premier procès-verbal de la SPRA, les artistes ont dû se réunir plusieurs fois (lacune dans les procès-verbaux) pour établir les nouveaux règlements et commencer l'admission des membres selon de nouvelles règles strictes d'épuration. Voir les observations sur les membres consignés dans la liste manuscrite adressée au Comité d'Instruction publique ( $c f$. note 9), dans les procès-verbaux, le Journal et sur le plat inférieur des six couvertures du Journal.

7 Journal, p. 4.

8 A plusieurs reprises les membres de la Société s'érigent en censeurs, mais les lois les empêchent de trop sévir: au moment de la dénonciation de Wicar, ils auraient brulé les tableaux de Fabre, Doyen et Ménageot s'ils ne s'étaient avisés qu'on ne peut détruire les biens appartenant à la Nation; dans l'affaire des graveurs receleurs d'images royalistes ils demandent de nommer des commissaires pour 's'informer des noms, demeures et qualités de ces traîtres.' Cette fois ce sont les termes même du code révolutionnaire qui les arrêtent.

9 A.N. $\mathrm{F}^{17} 1326$ doss 11, Nos 284-285. Au Comité d'Instruction Publique de la Convention Nationale sur la demande par lui faitte, à la Société libre des Arts, par sa lettre du Décadi de Brumaire de l'An deux de la République française une et indivisible. Je remercie James Leith de m'avoir communiqué ce précieux document.

10 Lapauze, Ibid., pp. 198-200.

11 Journal, p. 382-383. Le graveur Mathias fut-il vraiment seul responsable de l'association d'un titre fétiche de la Révolution - Ca ira - avec cette scène en effet un peu libre? Boilly mentait au sujet des titres: au début de la Révolution, il avait bel et bien peint un $A h$ ! ça ira, assorti du blason de la famille de Virieu, au bas d'un tableau de genre peint en camaïeu gris 'à l'imitation de l'estampe' (Musée de l'Hôtel Sandelin, Saint-Omer). L'inscription a-t-elle été ajoutée par allusion au chant révolutionnaire condamnant les aristocrates à la lanterne? Le sujet du tableau, une jeune mère mettant en garde ses enfants contre le chat qui guette leurs oiseaux pourrait le laisser croire.

12 Cette 'légende' d'une composition opportuniste exécutée 'par terreur et non par goût' a été récemment contestée par Susan L. Siegfried, sur le fait que Boilly a présenté son 24 avril (Acquittement de Marat) au Concours de l'An II, annoncé 
par David à la Société populaire et républicaine des arts le 25 avril, trois jours après la dénonciation lancée par Wicar; l'un n'empêche pas l'autre mais Siegfried ne tient pas compte de l'invitation de Boilly à venir vérifier à son atelier, dès le 28 avril, comment il avait exercé son pinceau d'une manière plus digne des Arts. 'Reconsidération du "Marat" de Boilly,' dans le catalogue de l'exposition Boilly, Un grand peintre français de la Révolution à la Restauration (Musée des beaux-arts de Lille, 1988-1989), pp. 6-10.

13 Lapauze, Ibid., p. 291. L'ortographe a été respecté dans les citations.

14 Lapauze, p. 297.

15 Journal, p. 30.

16 Journal, p. 31.

17 Journal, p. 31.

18 Journal, p. 32.

19 Le 23 août 1793 la Convention avait décrété la levée en masse, transformée en réquisition des hommes de 18 à 25 ans.

20 Journal, p. 45. C'est sans doute lui qui, dans la séance du 13 Pluviôse (1er février) de la SPRA a inspiré la 'demande qu'il ne soit délivré aucune carte aux artistes de 18 à 25 jusqu'à ce qu'ils prouvent les raisons d'exemption pour la réquisition,' Lapauze, Ibid., p. 219.

21 Journal, p. 42.

22 Journal, p. 58.

23 Journal, p. 47.

24 Journal, p. 43.

25 Journal, pp. 65-93.

26 Journal, p. 70.

27 Journal, p. 98.

28 Journal, p. 392.

29 Ces dates permettent de préciser celle de la fin de la rédaction du Journal après le 23 mai 1794 . 